\title{
Albergues y reubicación de los damnificados. Experiencias paceñas en gestión de crisis y vulnerabilidad
}

Refuges et relogement des sinistrés. Expériences pacéniennes dans la gestion de crise et de la vulnérabilité

Shelters and rehousing of disaster victims. Experiences of $\mathrm{La} P a z$ in crisis and vulnerability management

\section{Sébastien Hardy y Élodie Combaz}

\section{(2) OpenEdition}

Journals

Edición electrónica

URL: http://journals.openedition.org/bifea/2482

DOI: $10.4000 /$ bifea.2482

ISSN: 2076-5827

\section{Editor}

Institut Français d'Études Andines

\section{Edición impresa}

Fecha de publicación: 1 diciembre 2009

Paginación: 799-823

ISSN: 0303-7495

Referencia electrónica

Sébastien Hardy y Élodie Combaz, «Albergues y reubicación de los damnificados. Experiencias paceñas en gestión de crisis y vulnerabilidad », Bulletin de l'Institut français d'études andines [En línea], 38 (3) | 2009, Publicado el 01 junio 2010, consultado el 17 noviembre 2020. URL : http:// journals.openedition.org/bifea/2482 ; DOI : https://doi.org/10.4000/bifea.2482

Les contenus du Bulletin de l'Institut français d'études andines sont mis à disposition selon les termes de la licence Creative Commons Attribution - Pas d'Utilisation Commerciale - Pas de Modification 4.0 International. 


\title{
Albergues y reubicación de los damnificados. Experiencias paceñas en gestión de crisis y vulnerabilidad
}

\author{
Sébastien Hardy* \\ Élodie Combaz**
}

\section{Resumen}

Estos últimos años, los daños de origen natural que han afectado a la ciudad que sirve de sede al Gobierno boliviano condujeron las autoridades a aplicar nuevas políticas de gestión de los riesgos. Aquellas comenzaron a explorar a tientas la gestión de crisis teniendo en cuenta sus distintas fases: la emergencia, la recuperación y la reconstrucción. Las dos primeras fases les obligaron a pensar la organización de los auxilios y en particular el funcionamiento de albergues para los damnificados. La última fase les llevó a reflexionar sobre el riesgo y su gestión a largo plazo: en paralelo a la construcción de obras destinadas a resistir a las amenazas (violentas precipitaciones, crecidas, deslizamientos de terreno), establecieron mecanismos para retirar a las poblaciones damnificadas expuestas a las amenazas de origen natural reubicándolas en otras partes de la aglomeración de La Paz. Además del reconocimiento explícito de la impotencia de los gestores de los riesgos para reducir las amenazas, la política de reubicación demostró que tienen dificultades para concebir los riesgos en funcion de la identificación de elementos esenciales al funcionamiento de la aglomeración.

Palabras clave: riesgo, crisis, damnificado, albergue, reubicación, elemento esencial

* Institut de Recherche pour le Développement (IRD), UR 029, programa PACIVUR, Av. Hernando Siles 5290 esq. calle 7, CP 9214 Obrajes, La Paz, Bolivia. E-mail: sebastien.hardy@ird.fr

** Université de Provence-Institut de Recherche pour le Développement (IRD), UR 029, programa PACIVUR, Av. Hernando Siles 5290 esq. calle 7, CP 9214 Obrajes, La Paz, Bolivia. E-mail: elodie. combaz@etu.univ-provence.fr 


\title{
Refuges et relogement des sinistrés. Expériences pacéniennes dans la gestion de crise et de la vulnérabilité
}

\section{Résumé}

Ces dernières années, les dommages d'origine naturelle qui ont affecté la ville où siège le gouvernement bolivien ont conduit les autorités à mettre en œuvre de nouvelles politiques de gestion des risques. Ainsi, elles ont commencé à explorer par tâtonnement la gestion de crise en prenant en compte ses différentes phases : I'urgence, la récupération et la reconstruction. Les deux premières phases les ont obligées à penser l'organisation des secours, en particulier le fonctionnement de refuges pour les sinistrés. La dernière phase les a amenées à réfléchir au risque et à sa gestion à long terme : parallèlement à la construction d'ouvrages destinés à résister aux menaces (violentes précipitations, crues, glissements de terrain), elles ont mis en place des mécanismes pour soustraire les populations sinistrées à l'exposition aux menaces d'origine naturelle en les relogeant dans d'autres parties de l'agglomération de La Paz. Outre la reconnaissance explicite de l'impuissance des gestionnaires du risque à réduire les menaces, la politique de relogement a démontré qu'ils ont des difficultés à concevoir les risques eux-mêmes en fonction des enjeux.

Mots clés : risque, crise, sinistré, refuge, relogement, enjeu

\section{Shelters and rehousing of disaster victims. Experiences of La Paz in crisis and vulnerability management}

\begin{abstract}
In recent years, damages of natural origin have affected the town seat of the Bolivian government and these have led authorities to implement new policies of risk management. Thus, they have been seeking to manage crises by taking into account the various crisis phases: emergency, recovery and rebuilding. The first two phases have made them consider the organization of the assistance, in particular the management of shelters for the disaster victims. The last led them to think about risk and its long-term management: at the same time they have built works intended to withstand natural threats (violent precipitations, floods, landslides), and they have installed mechanisms to remove disaster victims from exposure to threats of natural origin by relocating them in other parts of metropolitan La Paz. In addition to the explicit recognition of the powerlessness of risk managers to reduce threats, the policy of relocation showed that they have difficulties in conceiving of risks in accord with what is at stake.
\end{abstract}

Key words: risk, crisis, disaster victim, shelter, relocation, strategic element

\section{INTRODUCTION}

El análisis de la base de datos DesInventar, que cataloga los eventos catastróficos en los países andinos, indica cómo las ciudades, y particularmente las capitales, son constantemente golpeadas por fenómenos de origen natural y/o antrópico1.

1 Ver en este volumen el artículo de R. D’Ercole, S. Hardy y J. Robert, pp. 433-465. 
Algunos de estos eventos originaron graves crisis que sirvieron de experiencia a las autoridades locales y nacionales para evaluar su capacidad en la gestión de las diferentes fases: la emergencia, la recuperación y la reconstrucción ( $\mathrm{D}^{\prime} E$ Ercole \& Metzger, 2004). Asimismo, dichos eventos nos permiten vislumbrar la evolución de la gestión de los riesgos — particularmente la de las vulnerabilidades-, tanto la efectuada por las autoridades encargadas, a través de las políticas y sus instrumentos como la que adoptan los individuos. De esta manera, resulta interesante informarse de las razones de ciertos desfases.

La aglomeración paceña no escapa a esta situación de conjunto. Es constantemente vulnerada y varios eventos han provocado unas verdaderas crisis. La ocasionada por la granizada del 19 de febrero de 20022 es considerada como referencia, poniendo las bases de un antes, de un durante y de un después. En efecto, el evento no había sido anticipado para nada. En consecuencia, la gestión de todas sus incidencias se hizo a tientas. Pero provocó una institucionalización de la gestión de crisis que las autoridades municipales han intentado mejorar a través de un retorno de experiencia pragmático después de cada nuevo evento. No obstante, la gestión de los damnificados parece ser una de las dimensiones que figuran entre las menos analizadas por las autoridades del Gobierno Municipal de La Paz (GMLP). Además continúan reflexionando muy poco en ello (Combaz, 2007), a pesar de que esta tiene consecuencias a mediano y a largo plazo sobre los riesgos y su gestión. En efecto, la gestión de los damnificados induce a una reflexión sobre la vulnerabilidad territorial (D’Ercole \& Metzger, 2009).

Durante la fase de emergencia, ayudar eficazmente a los damnificados implica reunirlos en ciertos lugares: los albergues. A partir del momento en que son rápidamente atendidos para recibir tratamientos sanitarios, psicológicos y alimentarios, la mayoría se recupera rápidamente del evento y se reintegra al curso normal de la vida. Otros, principalmente los que pierden su vivienda en el evento, necesitan una ayuda más duradera y se quedan en los albergues hasta encontrar donde reubicarse. La gestión de los albergues y de la reubicación están estrechamente ligadas. Son dos elementos importantes de una gestión de crisis y de los riesgos a largo plazo, en dos momentos diferentes (fase de recuperación y luego fase de reconstrucción). Su disfuncionamiento presagia una salida lenta de la crisis y el posible fracaso de la gestión de los riesgos a través de la reducción de la vulnerabilidad.

Por lo tanto, la reubicación de los damnificados es un elemento primordial de gestión de los riesgos. En efecto, la posición de resistencia a los fenómenos de origen natural a través de la construcción de obras de protección y de corrección ha demostrado, desde hace tiempo, su ineficiencia para disminuir la vulnerabilidad (Pigeon, 2005; Dauphiné \& Provitolo, 2007; Hardy, 2009). Por ende, reubicar adecuadamente a los damnificados permite intervenir significativamente sobre la vulnerabilidad de las poblaciones (Pelling, 2003). De este modo, las autoridades podrían reducir el riesgo reduciendo la exposición a las amenazas y la magnitud

2 Ver en este volumen el artículo de S. Hardy, pp. 501-514. 
de futuras crisis. No obstante, las operaciones de reubicación son bastante difíciles de efectuar, principalmente en los países del Sur. Necesitan medios importantes (financieros, técnicos, etc.) y una fuerte voluntad política, ya que implican una importante movilización de las autoridades en estrecha relación con las necesidades de los beneficiarios, para que la oferta responda a las expectativas de los damnificados (Revet, 2007). Su éxito supone también un seguimiento, en el tiempo, de los damnificados reubicados. Considerar las interacciones entre estos factores permite seguir el camino de una gestión más eficaz de los riesgos.

El análisis de dos crisis en la municipalidad paceña - la una provocada por la granizada del 19 de febrero de 2002 y la otra por el deslizamiento de terreno en el barrio de Llojeta el 4 de marzo de 2003- muestra que puede haber una real movilización de las autoridades locales para manejarlas, y en particular para reubicar a los damnificados. Esto nos lleva a interrogarnos sobre la reducción de la vulnerabilidad de los reubicados a largo plazo. Por esta razón el análisis de la reubicación de los damnificados permite al mismo tiempo comprender mejor la gestión de crisis en sus fases de emergencia y de recuperación, así como las políticas de gestión de los riesgos en la fase de reconstrucción. Por lo tanto, al ocuparse de las poblaciones damnificadas y de su reubicación, también se toma en cuenta su vulnerabilidad. Para lograrlo, presentaremos en un primer tiempo la gestión de los damnificados que hayan perdido su vivienda en la granizada del 19 de febrero de 2002, en sus fases sucesivas de emergencia, de recuperación y de reconstrucción para establecer los diferentes aspectos de una experiencia que ha servido de marco de referencia. Luego nos dedicaremos a las evoluciones de esta experiencia respecto a nuevos eventos que hayan afectado a la aglomeración de La Paz. Estos dos tiempos ofrecen la posibilidad de reflexionar sobre la gestión de las crisis puesta en marcha por las autoridades y sobre los ajustes que los individuos aportan separadamente, para mostrar que estas diferencias son signos de la importancia mal aprehendida de ciertos elementos.

\section{LA GRANIZADA DE FEBRERO DE 2002: PRIMERA EXPERIENCIA DE REUBICACIÓN DE DAMNIFICADOS}

\subsection{Los albergues en la gestión de crisis}

\section{1. 1. Albergues «espontáneos» en fase de emergencia}

Las inundaciones y los deslizamientos de terreno que se presentaron junto con la granizada del 19 de febrero de 2002 dañaron fuertemente las estructuras, y la vivienda, muy en particular. Asimismo, desde la fase de emergencia, las autoridades encargadas procuraron dar auxilio a muchas familias que perdieron su casa. Sandrine Revet define este momento como

«particularmente corto [marcado] [...] por sus características operativas. Se trata del tiempo durante el cual se llevan a cabo las acciones de salvamento y de evacuación de los habitantes fuera de la zona afectada por el desastre. 
En esta fase durante la cual el peligro se ha alejado [...], las autoridades recobran el control de la situación» (Revet, 2007: 145).

En efecto, durante la granizada, los mismos habitantes tuvieron muchas veces que dar respuestas inmediatas a sus dificultades, poniéndose a cubierto en casa de los vecinos, en lugares públicos o considerados como tales. En cambio, para las autoridades, la entrada en fase de emergencia consistió en brindarles ayuda lo más rápidamente posible (distribución de frazadas y de alimentos, primeros auxilios, etc.).

La cartografía de los casos de emergencia que fueron tratados por los servicios municipales3 muestra que gran parte del espacio de La Paz fue afectado por la granizada4. Asimismo, en un primer tiempo, las autoridades buscaron reunir a los damnificados en un número limitado de lugares, cerca de las zonas afectadas. Las escuelas, las salas comunitarias de barrio y las iglesias — donde muchos damnificados se habían refugiado espontáneamente al comienzo de la emergencia - fueron designadas como albergues. Estos, numerosos y dispersos, reunieron tanto a residentes del barrio como a damnificados presentes en el barrio en el momento del evento y facilitaron a las autoridades la asistencia a los damnificados: primeros auxilios médicos y distribución de frazadas.

Durante esta fase de emergencia, las autoridades ayudaron lo mejor que pudieron a los damnificados. Para ello, tuvieron que tomar en cuenta la temporalidad del evento: la granizada del 19 de febrero de 2002 se produjo en plena tarde (14:20 a 15:45), un día de semana. Por lo tanto, en el momento en que se produjo el evento, las familias estaban por lo general separadas: los padres en su lugar de actividad profesional, los hijos en su lugar de estudios o de recreo, etc. La resultante de la temporalidad se encontró en todos los primeros albergues de emergencia: los padres estaban separados de sus hijos. Por lo tanto, las autoridades tuvieron que buscar a los miembros de las familias para reconstituirlas $y$, con este fin, registraron a los damnificados y sus necesidades. Este primer registro de emergencia dio una gama de 800 a 850 damnificados y permitió realizar una cartografía de la distribución de los damnificados, a escala media (fig. 1). Estos se agrupaban en cuatro macrodistritos, que constituían los espacios más dañados: Centro, Sur, Cotahuma y Periférica.

La intensidad de las precipitaciones se concentró en el macrodistrito Centro. El hecho que haya experimentado mayores daños tiene su explicación en la temporalidad del evento. De día, el espacio Centro reúne empleos formales (administración del Estado, del departamento y de la municipalidad; sedes del Banco Central y de los principales bancos bolivianos, etc.) y empleos informales (puestos de venta de alimentos y bebidas, lustrabotas, etc.). La población converge hacia el centro generando flujos intensos. El evento tuvo lugar en plena tarde un día de semana por lo cual los damnificados fueron numerosos.

La fuerte presencia de damnificados en el macrodistrito Sur se debe a la concentración de las aguas de escorrentía superficial en la salida natural que es el

\footnotetext{
3 Se trata de todas las intervenciones que los servicios municipales han efectuado para acudir en ayuda de la población damnificada por la granizada.

4 Ver en este volumen, el artículo de S. Hardy, pp. 501-514.
} 


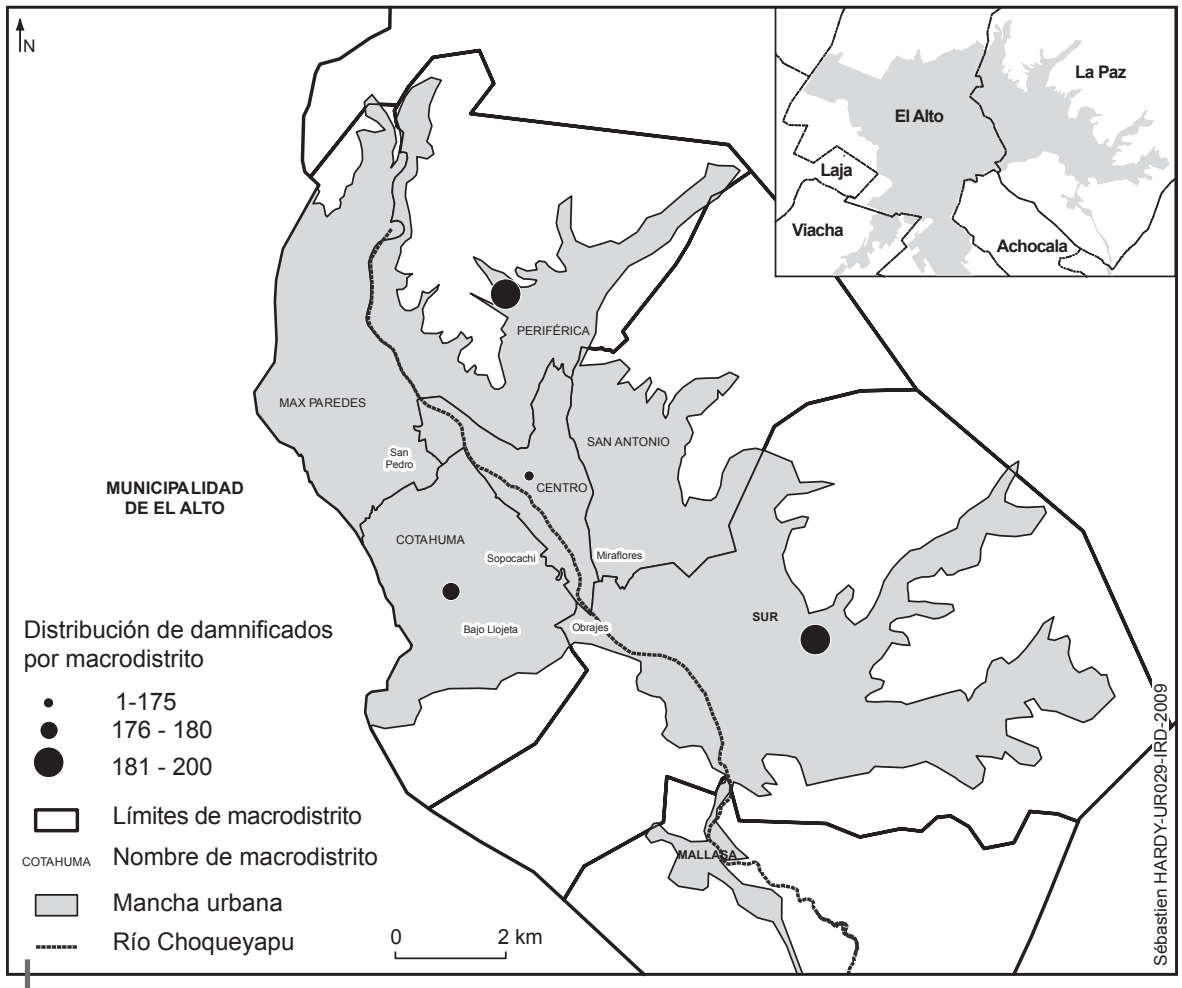

Figura 1 - La distribución de los damnificados en los albergues espontáneos por macrodistrito, fase de emergencia, en febrero de 2002

torrente Choqueyapu. Escurriéndose dentro de desagües subterráneos en la parte central de la ciudad, el Choqueyapu está canalizado ya cuando atraviesa el sur de la ciudad. El 19 de febrero de 2002 su crecida provocó la inundación de muchos barrios residenciales acomodados, así como cortes de vías de comunicación que aislaron a unos barrios de otros5, causando muchos damnificados.

Por último, la importante cifra de damnificados de los macrodistritos Cotahuma y Periférica se debe a las precipitaciones que activaron localmente los deslizamientos de tierra, los cuales provocaron la destrucción de viviendas generalmente bastante mal construidas por familias pobres (estructuras de soporte débiles, materiales de mala calidad, etc.).

Esta repartición espacial de los daños justifica la variedad de perfiles de los damnificados. Paradójicamente, casi no se diferenciaron durante la fase de emergencia. Para salir rápidamente de ésta, todos necesitaban una asistencia a la cual tuvieron acceso en los albergues espontáneos donde se organizaron los

5 Ver en este volumen el artículo de J. Núñez y F. Demoraes, pp. 827-848. 
primeros auxilios. Para las autoridades encargadas, los damnificados formaban en ese momento de emergencia una sola población de víctimas que manejaron sin distinción.

Muchos testimonios señalaron la profunda desorganización de los auxilios de emergencia. Las autoridades no estaban preparadas para manejar una fase de emergencia. A pesar de las anteriores crisis, ningún lugar había sido identificado como albergue potencial; para brindar asistencia, no se había previsto ningún procedimiento particular con un protocolo de acciones a seguir, y tampoco se había preparado a las poblaciones. La tormenta reveló por lo tanto la profunda falta de preparación de las autoridades, principalmente por la falta de anticipación para este tipo de evento, lo que generó la crisis.

No obstante, ante la amplitud de los problemas de todo tipo, las autoridades trataron de organizarse rápidamente para aportar soluciones, sobre todo para reubicar a los damnificados.

\section{1. 2. Albergues temporales en espera de soluciones: de la fase de emergencia a la de recuperación}

Al final de la fase de emergencia, parte de los damnificados pudo regresar a su domicilio. En efecto, así los más acomodados como los menos afectados se encargaron rápidamente de su propio destino. De suerte que buena parte de los damnificados de los macrodistritos Centro y Sur desaparecieron rápidamente del escenario de la emergencia. Por lo tanto, solo quedaron en los albergues espontáneos de la fase de emergencia los damnificados cuya situación era más precaria. Estas situaciones dispares explican la dificultad para estimar el número de noches que pasaron los damnificados en los albergues espontáneos: los testimonios varían de una o dos noches hasta dos semanas.

Con el retorno a la normalidad de una parte de los damnificados, entonces comenzó una segunda fase para las autoridades encargadas de la gestión de la crisis: la recuperación (o rehabilitación). Su organización de la gestión de crisis tuvo que aumentar en capacidad para resolver nuevos problemas, como el de los damnificados que perdieron sus viviendas en el evento y para los cuales continuaba la crisis. A fin de que mejoraran las condiciones de asistencia, las autoridades trataron de reunir a los damnificados sin vivienda, los cuales se encontraban muy dispersos en los albergues espontáneos. De manera que los transfirieron hacia dos estructuras elegidas desde la fase de emergencia, a partir del primer registro establecido en los albergues espontáneos. Estos albergues temporales —el Coliseo Cerrado Julio Borelli Viterito y el Coliseo de la Universidad Mayor de San Andrés - fueron elegidos por sus características, lo que hizo que la intervención de los auxilios fuera más fácil durante esta segunda fase de gestión de crisis6.

6 Hubieron hasta 700 personas refugiadas en el Coliseo Cerrado Julio Borelli Viterito y 125 en el Coliseo de la UMSA. 
Se trata de estadios cubiertos, vastos, próximos uno del otro, en una zona de la ciudad muy accesible (fig. 2). La implementación de los servicios de auxilio mejoró sensiblemente. El espacio total de los albergues fue dividido en celdas de $3 \mathrm{~m}^{2}$ y a cada familia se le atribuyó una, sin que importe su composición. De la suerte las autoridades estuvieron más directamente en contacto con los damnificados a fin de acompañarlos en su recuperación.

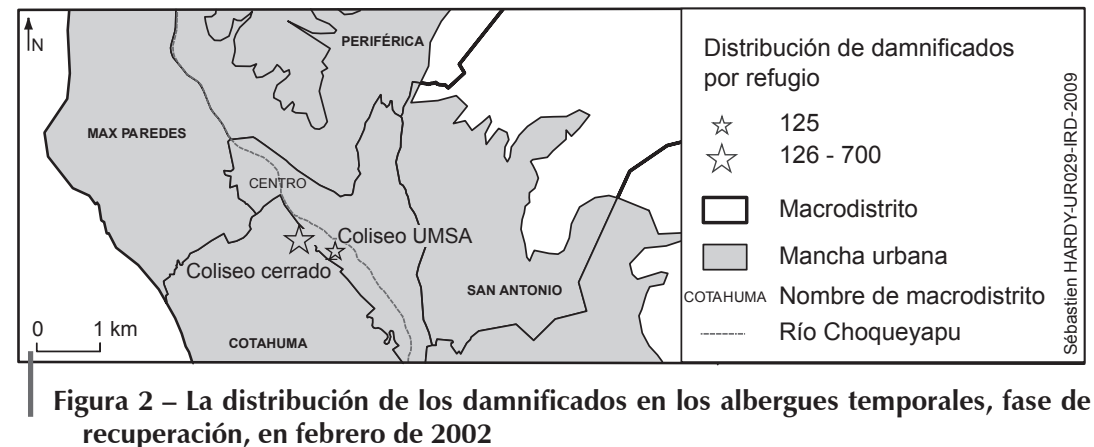

A pesar de las sensibles mejoras de las condiciones de albergue, otros problemas surgieron. Después de la transferencia, un nuevo registro realizado en los albergues temporales mostró que habían llegado nuevas personas, aumentando así la cifra de damnificados sin que las autoridades hubieran sido capaces de determinar si se trataba de verdaderos damnificados de la granizada, o simplemente de personas que habían aprovechado la situación para ser atendidas. Luego, continuamente se señalaron problemas de orden público lo que creó tensiones. Tanto las autoridades como los damnificados atribuyeron aquellos al alcoholismo y al comportamiento violento de algunos individuos. Asimismo, contribuyeron a esta situación la promiscuidad y las condiciones generales de reubicación (habitantes pobres, etc.).

En estas condiciones, muchos damnificados no lograron resolver sus problemas por sí mismos y en primer lugar su reubicación. Sin embargo la magnitud del evento les fue favorable ya que facilitó el compromiso de las autoridades de brindarles ayuda.

Las autoridades bolivianas, principalmente las del municipio de La Paz, fueron severamente criticadas por su falta de anticipación de los problemas. Si bien los desastres son frecuentes en Bolivia y participan en mantener al país en la pobreza, el país todavía no dispone de un fondo nacional específico dedicado a la resolución de las crisis relacionadas con los desastres. Pero en febrero de 2002, la magnitud de los daños —y el hecho que La Paz sea la sede del gobierno, de las instituciones internacionales y de las principales actividades terciarias del paíspermitió movilizar tanto la solidaridad nacional como internacional. Las ayudas recibidas (estimadas en 25,4 millones \$ US) han permitido encontrar soluciones de reubicación para los damnificados (Villegas, 2002: 32). Así, las autoridades 
(nacionales y de la municipalidad), se dieron cuenta que jugaban su credibilidad en la resolución de esta crisis.

\section{2. De albergues temporales a alojamientos definitivos: la fase de reconstrucción}

\section{2. 1. Los actores de las reubicaciones}

Las autoridades nacionales, que fueron severamente criticadas por su falta de preparación frente a los desastres de origen natural, tomaron rapídamente la decisión de reubicar a los damnificados iniciando así la fase de reconstrucción. Sin embargo, a falta de un protocolo previo que determinara las condiciones de una reubicación en caso de un desastre de origen natural, las autoridades tuvieron que crearlo, dilatando los plazos para lograr una reubicación efectiva de los damnificados.

El establecimiento de este protocolo ha planteado algunas preguntas respecto a la reubicación de los damnificados:

- «¿Quienes son los beneficiarios?» es una interrogante que ha permitido establecer criterios de selección de los damnificados;

- «żcon qué fondos?» ha permitido identificar y movilizar los actores capaces de financiar la reubicación;

- «¿dónde reubicar?» ha puesto en evidencia la falta de terrenos construibles no expuestos a las amenazas de origen natural dentro del espacio del municipio de La Paz.

Este hecho obligó a buscar y a negociar la adquisición de terrenos en los municipios vecinos, y también convencer a los beneficiarios para que aceptaran desplazarse fuera de La Paz (con las consecuentes molestias por el alejamiento de la escuela de los niños, de los lugares de trabajo, etc.).

Para responder a estas preguntas, las autoridades crearon un Comité de reubicación que integró a todos los actores: damnificados, autoridades del GMLP, autoridades públicas nacionales como el ministerio de Obras Públicas, Servicios y Vivienda, actores privados como las ONG, empresas de construcción y organismos financieros. Todos estos actores no intervinieron al mismo tiempo, lo que permite distinguir dos niveles de funcionamiento:

- un primer nivel entre damnificados y autoridades del GMLP (1), que se organizó al interior de la Comisión de damnificados propiamente dicho. Estos actores determinaron los beneficiarios de una reubicación y constituyeron sus expedientes, así como los compromisos entre reubicador y reubicado.

- un segundo nivel se estableció entre las autoridades del GMLP y el resto de los actores nacionales e internacionales (2), para buscar y comprar parcelas destinadas a la reubicación, y para decidir sobre la reasignación de los terrenos golpeados por el desastre. 
La cantidad de preguntas que hubo que contestar, vinculada al gran número de actores con prerrogativas, unas veces complementarias otras veces imbricadas, explica la demora del protocolo de reubicación. Si se toma el ejemplo de la Comisión de damnificados en el primer nivel de funcionamiento (1), se ve que esta implicó por una parte a cuatro actores distintos provenientes del GMLP (Dirección jurídica, Oficialía de gestión territorial, Oficialía técnica y alcaldías de macrodistritos), y por otra parte a los damnificados. Unas reuniones semanales brindaron a estos actores la ocasión de enterarse juntos de los expedientes de los damnificados, lo que facilitó la elaboración de los expedientes de reubicación.

El director de este primer nivel de la Comisión de damnificados, proveniente de la Dirección jurídica del GMLP, era solicitado por el damnificado. Comenzaba su trabajo determinando la categoría a la cual pertenecía el damnificado: un propietario legalmente inscrito en el catastro que ha perdido su vivienda; un inquilino cuya vivienda fue destruida por la granizada. Por lo tanto, ser damnificado no era una condición suficiente para beneficiarse de la operación de reubicación definitiva.

La resolución del caso de los damnificados inquilinos fue rápida. Las autoridades decidieron darles una ayuda financiera para su realojamiento. En el caso de los propietarios cuya vivienda fue destruida por la granizada, se inició un proceso de reubicación.

No fue fácil establecer la categorización de los damnificados. Muchos de ellos habían perdido, con su vivienda, las actas legales de propiedad. En este caso, el Director de la Comisión de damnificados tuvo que recurrir a la Oficialía de gestión territorial para encontrar la inscripción del propietario en el catastro, y verificar que efectivamente ocupaba esa vivienda en el momento del desastre y que no era propietario de otra vivienda. A veces esta etapa fue muy larga ya que las búsquedas resultaron complicadas cuando se trataba por ejemplo de una vivienda heredada y que las familias no habían hecho una declaración de sucesión.

Después de esta etapa de categorización, el Departamento técnico del GMLP debía realizar, a solicitud del damnificado, una inspección geotécnica del terreno donde la vivienda había sido destruida para confirmar su carácter inconstruible.

A partir de todos esos datos, el Director de la Comisión podía establecer un compromiso entre el reubicado y el reubicador: un intercambio de actas de propiedad a través del cual la municipalidad de La Paz obtenía la propiedad destruida a fin de evitar futuras reocupaciones.

La cooperación entre los actores de este primer nivel de la Comisión de damnificados era necesaria para el buen avance de los expedientes. No obstante, el damnificado siempre fue intermediario entre los diferentes actores del GMLP. Al ignorar ciertos servicios y ciertos procedimientos, sus titubeos administrativos prolongaron el tiempo de tratamiento de los expedientes. Este hecho conduce a interrogarse sobre la organización y el funcionamiento del primer nivel de la Comisión.

En el segundo nivel de funcionamiento (2), los actores nacionales de la Comisión propusieron al GMLP terrenos dotados de viviendas para los damnificados a 
cambio de completar los expedientes. De hecho el ministerio de Obras Públicas, Servicios y Vivienda fue quien administró los fondos de emergencia atribuidos por el Estado y procedió, con los organismos financieros, a una licitación ante constructores privados para comprar parcelas construidas.

Entre estos actores y estos dos niveles de funcionamiento, a veces las interacciones fueron más complicadas de lo que prefiguraba la organización prevista. Por ejemplo, la Comisión de damnificados del GMLP había entregado al ministerio de Vivienda una lista de los damnificados potencialmente beneficiarios de una reubicación. Pero el Ministerio no siempre la había aprobado. Para establecer esta lista, el primer nivel de la Comisión de los damnificados había privilegiado los criterios legales y técnicos (título de propiedad y terreno inconstruible), mientras que el ministerio de Vivienda solo había prestado atención a los criterios sociales y económicos (números de miembros por familia y sus recursos). Esta situación creó tensiones puntuales entre actores: la cooperación entre actores no fue cuestionada sino sus formas, y por ende, la definición precisa de las prerrogativas de cada uno. Esta ausencia de precisión provocó imbricaciones de competencias en detrimento de una complementariedad más eficaz. Por ejemplo, ipor qué el GMLP estableció una lista de beneficiarios si el ministerio de Vivienda tenía otra en función de criterios diferentes? Estos defectos de articulación pueden explicar también la elección de los lugares de reubicación de los damnificados, los mismos que respondían muy parcialmente a sus necesidades reales.

\section{2. 2. Los lugares de reubicación definitiva}

Tras el desastre del 19 de febrero de 2002, 166 familias se beneficiaron de una operación de reubicación definitiva que se operó en varios lugares, los cuales estaban situados en el municipio vecino de El Alto, en la periferia de La Paz. Se trata de los barrios Cristal I, Cristal II y Palermo7 (fig. 3).

Estos tres barrios de reubicación de los damnificados presentan globalmente las mismas características. Están bastante alejados del lugar de vivienda de origen de los damnificados. Por otro lado, se encuentran aproximadamente a una hora y media en bus desde el centro de La Paz, insertos en zonas desfavorecidas de El Alto. Por último, presentan una disposición muy similar que no toma en cuenta el tamaño de las familias: parcelas de igual superficie, yuxtapuestas unas con otras; las casas construidas son todas iguales.

Cristal I constituye el principal barrio de reubicación definitiva de los damnificados. Ha acogido a 68 familias damnificadas por la granizada del 19 de febrero de 2002, más 10 familias damnificadas por el deslizamiento de terreno de Cotahuma de 19948. Pero este barrio no había sido concebido para acoger a damnificados.

\footnotetext{
7 A veces también conocido bajo el nombre de Nueva Tilata 3.

8 Las autoridades aprovecharon la operación de reubicación iniciada por la granizada de febrero de 2002 para reubicar a los damnificados de otras catástrofes para los cuales no se habían encontrado soluciones hasta entonces. Antes de su reubicación definitiva, estos damnificados vivían en campamentos dispersos en todo el municipio de La Paz.
} 


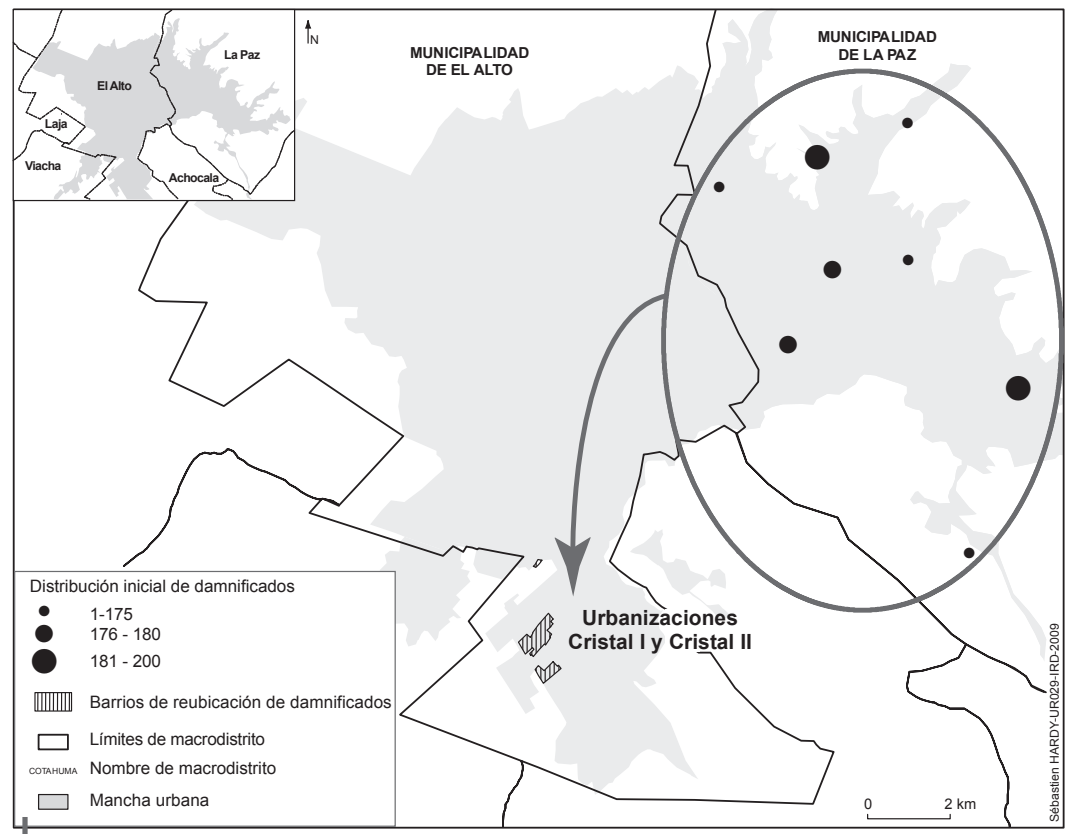

Figura 3 - Los barrios de reubicación de los damnificados

Cuando estos se trasladaron ya vivían familias allí — esencialmente constituidas por ex mineros provenientes de Colquiri y de Caracoles-. Las parcelas construidas que todavía estaban disponibles fueron compradas a 3000 \$ US por el ministerio de Vivienda en beneficio de los damnificados.

Cristal II está construido sobre el mismo modelo que Cristal I. Mayormente ocupado por familias de policías beneficiarias del Programa Nacional de Vivienda Social (PNVS), el barrio ha acogido a 73 familias beneficiarias de la reubicación. Se trata de familias que han perdido su vivienda en diversos desastres y para las cuales las autoridades no habían encontrado solución de reubicación antes del evento de 2002: 44 familias damnificadas por el deslizamiento de terreno de Kupichico, y 6 familias damnificadas por el evento de Kupini de 1999. Las autoridades también reubicaron allí a 73 familias damnificadas por el evento de Llojeta del 4 de marzo de 2003.

El barrio Palermo es el más alejado en distancia y en tiempo del centro de La Paz. Su disposición es bastante diferente de los barrios Cristal I y Cristal II, con una yuxtaposición de tipos de estructura: de un solo piso y de dos pisos. Las propiedades también parecen mejor mantenidas por los ocupantes iniciales (casas cercadas, etc.) beneficiarios del PNVS. A partir del 2002, 15 familias damnificadas por la granizada del 19 de febrero de 2002 fueron reubicadas allí. Posteriormente se les han unido 50 familias damnificadas por el deslizamiento de terreno de Llojeta del 4 de marzo de 2003. 
La operación de reubicación luego de la granizada, en los tres barrios ya mencionados, se concretó a partir del 18 de marzo de 2002, fecha en la cual camiones del GMLP comenzaron a trasladar hacia su nueva vivienda a las familias - y los bienes que les habían dado en los albergues temporales (Villegas, 2002: 48)—. A lo largo del año 2002, se han beneficiado con la ayuda de diferentes ONG en sus barrios de reubicación.

La repartición de los damnificados en los barrios no respondió a ninguna lógica socio-espacial sino simplemente a la cronología de tratamiento de los expedientes. El barrio Cristal II es el que ha recibido más damnificados aunque paradójicamente ninguno era de la granizada de 2002. La operación de reubicación fue sobre todo la oportunidad para las autoridades nacionales y municipales de resolver las situaciones de los damnificados de otros desastres de menor magnitud, para las cuales las soluciones institucionales y financieras no existían.

\section{CONSIDERACIÓN DEL BALANCE DE LA CRISIS DE 2002 EN CRISIS POSTERIORES}

\section{1. La granizada y sus consecuencias institucionales para el municipio de La Paz}

La granizada del 19 de febrero de 2002 marcó al mismo tiempo el punto de partida del aprendizaje de la gestión de una crisis y de una reflexión sobre la reducción de los riesgos mediante la reubicación. Los servicios del GMLP, organizados en comisiones (salud, albergues, servicio social, alimentación y nutrición, apoyo psicológico), intervinieron para administrar los albergues, distribuir las donaciones, seleccionar a los beneficiarios de realojamiento, etc. Este aprendizaje condujo a una resolución municipal9 destinada a crear un nuevo organismo municipal: el Comité de Operación de Emergencia (COE).

Esta estructura evolucionó. Por ejemplo, el 26 de agosto de 2006, mediante la resolución municipal n. ${ }^{\circ}$ 0480/2006, se implementó la descentralización del COE para facilitar las sinergias entre la escala municipal y la del barrio, gracias a la creación de los Comités de Operación de Emergencia de Macrodistritos (COEM) y de los Comités de Operación de Emergencia de Barrios (COEB)10. Estos nuevos niveles se encargaron principalmente de identificar albergues potenciales en su área de competencia (macrodistrito y barrio), a fin de mejorar la preparación para una gestión de crisis.

Si se presta atención al funcionamiento de las estructuras creadas, se observa que las informaciones circulan bastante bien entre el COE y el COEM. En cambio, parece más difícil sensibilizar al barrio, principalmente sobre el tema de los albergues. En efecto, los presidentes de barrio son los responsables de la identificación de los

9 Resolución municipal n. ${ }^{\circ}$ 0344/2002 del 29 de noviembre de 2002.

10 Resolución municipal n. ${ }^{\circ}$ 0480/2006 del 26 de agosto de 2006. 
albergues. Pero, generalmente están acaparados por otras tareas consideradas más urgentes por los habitantes a quienes les deben su mandato.

No deja de ser cierto que el COE, con su estructura piramidal implementada retrospectivamente después de la experiencia de la crisis del 19 de febrero de 2002, es el organismo municipal mejor preparado en materia de gestión de crisis a nivel de Bolivia. Por otro lado, las autoridades municipales no han cesado de mejorarlo después de cada nueva crisis.

\section{2. Alto Llojeta: modificaciones de los procedimientos de gestión de crisis por aprendizaje}

El 4 de marzo de 2003, el COE, que fue designado como el operador de la gestión de crisis, fue puesto a prueba con ocasión de un nuevo desastre: un deslizamiento de terreno en el barrio de Alto Llojeta, ubicado en las laderas oeste del municipio paceño (O'Hare \& Rivas, 2005). Más arriba de la ladera Alto Llojeta, ocurrieron dos derrumbes que provocaron un movimiento de terreno sobre gran parte de la ladera. Este evento privó de vivienda a 87 hogares de la zona y por su magnitud fue declarado catástrofe nacional.

Los damnificados del deslizamiento de terreno de Alto Llojeta eran familias de fabricantes de ladrillos, instaladas en el lugar donde se abastecían de la materia prima. Por lo tanto, reubicar definitivamente a estos damnificados resultó ser una operación complicada. Su traslado hacia otro terreno implicaba la pérdida de su empleo. Asimismo, el interés de este evento reside en la observación de la gestión de la crisis por parte de las autoridades, en función de la experiencia adquirida en 2002: evolución de la toma en consideración de la situación inicial de las familias y definición de nuevos criterios de reubicación para evitar los defectos anteriores. Por eso este evento fue una ocasión para que las autoridades municipales evaluaran la utilidad del $\mathrm{COE}$, en particular en términos de reubicación en fase de emergencia, y luego en fase de reconstrucción.

En comparación con febrero de 2002, la gestión de crisis en fase de emergencia en marzo de 2003 evolucionó en tres aspectos: los lugares elegidos para la reubicación temporal; los tipos de albergues elegidos; la evolución de los modos de organización. Un contexto diferente también ha ayudado a estas evoluciones: en 2003, las zonas afectadas son localizadas con más precisión. Por lo tanto, no hay inmovilización casi generalizada del conjunto de la aglomeración como en 2002, y los daños son menores. Así, es más fácil para las autoridades organizarse para enfrentarse con la emergencia. Los procedimientos desarrollados durante la fase de emergencia y de recuperación fueron los que se aplicaron en 2002, corregidos en algunas de sus imperfecciones.

Durante la crisis de febrero de 2002, los damnificados habían sido transferidos de albergue en albergue, antes de ser reubicados muchos meses más tarde. En marzo de 2003, los damnificados fueron directamente transferidos hacia tres campamentos situados cerca de la zona del impacto: Raúl Salmón, Alpacoma y 
Llojeta El Vergel. El objetivo era reducir el trauma psicológico y la desorganización de las actividades cotidianas. Esta reubicación provisional cercana a la zona de impacto en campamentos bajo carpas proporcionadas por la Defensa civil boliviana, en lugar de los albergues construidos, respondía a una necesidad de flexibilidad. En efecto, existen normas internacionales que dictan la organización de los albergues: una estructura sólida, una superficie mínima por damnificado, en las que se deben respetar densidades y condiciones sanitarias estrictas. Es bastante difícil encontrar albergues que a nivel local permitan respetar estas normas. En estas condiciones, es más fácil instalar un campamento a fin de aplicar las normas internacionales: carpas con orientación norte-sur, la entrada de las carpas hacia el centro del campamento y cada carpa situada como mínimo a 6 metros de distancia de los depósitos de basura.

Al reunir a los damnificados en campamentos cercanos al lugar del desastre, las autoridades procuraron evitar también la intrusión de los no damnificados quienes simplemente habrían buscado ser atendidos, como ocurrió en febrero de 2002. En efecto, es más fácil vigilar las idas y vueltas de personas en tres lugares excentrados sobre todo cuando los damnificados, residentes de los lugares dañados, se conocen. Por lo tanto, las autoridades eligieron lugares no expuestos a la amenaza, cerca del lugar del desastre, de acceso cómodo y de fácil conexión a los servicios básicos de agua potable, electricidad y teléfono.

Por otro lado, un campamento limita la dependencia hacia políticas de asistencia. Para su funcionamiento, los damnificados son mucho más requeridos: limpieza, preparación de las comidas y seguridad. Las condiciones de reubicación provisionales, menos cómodas, tienden a reducir la duración de permanencia de los damnificados. Durante la crisis de febrero de 2002, cuando las autoridades invitaron a los damnificados del Coliseo de la Universidad Mayor de San Andrés y del Coliseo Cerrado a desalojar los lugares para ser transferidos hacia una nueva vivienda, algunos protestaron enérgicamente rehusándose incluso a partir. Las autoridades identificaron este movimiento como una reacción de los damnificados frente a la incertidumbre de una nueva vida en la cual les tocaría hacerse cargo de sí mismos. Por ello, en los campamentos de Llojeta, las autoridades solicitaron una mayor participación de los damnificados para evitar una situación de dependencia. Además, habían observado, en febrero de 2002, que los damnificados eran menos vulnerables de lo que anunciaba la situación de emergencia. En efecto, tenían recursos y capacidades que movilizaron, para ellos mismos y para la colectividad, como por ejemplo en la organización de los albergues temporales: se instalaron cocinas colectivas administradas por los damnificados, las cuales eran abastecidas gracias a donaciones alimentarias por parte de las autoridades. También se organizaron actividades para los niños, con ayuda de las autoridades: transporte diario a las escuelas y cuidado de los más pequeños durante la ausencia de los padres. En los campamentos instalados en marzo de 2003, las autoridades aprovecharon de manera más sistemática estas habilidades de los damnificados identificadas durante la crisis de febrero de 2002. También la elección por las autoridades municipales del campamento en marzo 
de 2003 resultó del aprendizaje de las dificultades encontradas durante la gestión de crisis de febrero de 2002 (Combaz, 2007).

En cuanto a la fase de reconstrucción, los aprendizajes de 2002 son menos evidentes. Indudablemente la gestión de esta nueva crisis demostró la eficiencia de la Comisión de damnificados que logró tratar con rapidez los expedientes de reubicación. Paralelamente, las autoridades incitaron los damnificados a encontrar ellos mismos soluciones temporales de reubicación —o auto-refugios- en el domicilio de parientes o amigos, para mejorar lo más pronto posible la recuperación en la espera de una reubicación definitiva. Pero la fase de reconstrucción no permitió reconsiderar las políticas de gestión de los riesgos, cuando las primeras fallas ya se estaban produciendo.

Por lo tanto, las autoridades municipales aprendieron la necesidad de pensar, crisis tras crisis, en las medidas de gestión más adaptadas, haciendo evolucionar los procedimientos en fase de emergencia y de recuperación. Por ejemplo, la evolución de los lugares de los albergues fue el fruto de la voluntad de preservar la cercanía: esta indujo la evolución de las estructuras de refugio, lo que provocó una descentralización de las operaciones e implicó una necesidad más importante de las competencias de los damnificados para hacer funcionar los campamentos. Esta evolución introdujo nuevas prácticas que todavía están a prueba, a partir de las cuales se acordará mejorar progresivamente los procedimientos de gestión de las fases de emergencia y de recuperación.

\section{3. Las preguntas planteadas por la gestión del evento de Alto Llojeta}

Los ajustes efectuados durante la gestión de crisis de Alto Llojeta parecen responder a la necesidad de evitar los problemas observados durante la crisis de febrero de 2002. Sin embargo, algunos, como la invitación al auto-refugio en fase de recuperación, no parecen muy pertinentes. Esto implicó la dispersión de los damnificados en toda la aglomeración dificultando el seguimiento (sanitario, psicológico y constitución de expedientes) de los damnificados por parte de las autoridades hasta el momento de la reubicación definitiva.

El evento de Alto Llojeta pone de manifiesto que la declaración de desastre nacional ha sido nuevamente un elemento clave para la reconstrucción. Ha permitido a las autoridades municipales contar con medios, principalmente financieros, y efectuar, como en febrero de 2002, la reubicación de los damnificados en El Alto, siguiendo los mismos procedimientos: 50 familias en el barrio Palermo y 37 familias en el de Cristal II. Por lo tanto, la reconstrucción se asemejó a la de 2002, a pesar de los problemas que ya empezaban a observarse. No obstante, resultó más fácil que durante los eventos que no fueron acompañados de una declaración de catástrofe nacional (Combaz, 2007).

Sin embargo, la reubicación definitiva de los damnificados del evento de marzo 2003 resultó menos evidente de manejar para las autoridades municipales. Estas se dieron cuenta de lo difícil que es impedir la posible reinstalación de los 
damnificados en el lugar que les proporciona recursos. Después de algunos meses de tergiversaciones, se vieron obligadas a adoptar una medida más pragmática aceptando que los obreros reinstalaran sus fábricas en el sitio de Alto Llojeta y realizaran migraciones pendulares entre El Alto y La Paz para trabajar ahí. A cambio de esto, las autoridades pidieron ser liberadas lo más posible de la responsabilidad respecto a la exposición de las poblaciones al daño potencial, sin realmente haber obtenido garantías.

\section{3. ¿CUÁL ES LA DURABILIDAD DE LA REUBICACIÓN? LA GESTIÓN DE LA VULNERABILIDAD Y DEL RIESGO}

\section{1. El costo del transporte, un factor negativo para los damnificados reubicados}

En rigor resulta difícil establecer, algunos años después, un balance exhaustivo de las operaciones de reubicación de los damnificados de la granizada del 19 de febrero de 2002 y de los del deslizamiento de terreno de Alto Llojeta del 4 de marzo de 2003. Sin embargo, la visita a los barrios de reubicación y las entrevistas con las poblaciones proporcionan materia para interrogarse sobre su éxito. Al visitar los barrios con frecuencia se ha visto viviendas vacías que parecen estar abandonadas (fig. 4); además las entrevistas con varios habitantes (Combaz, 2007) lo han confirmado y han permitido entender algunas de las razones.

Los damnificados reubicados dependen en su mayoría de ingresos provenientes de actividades formales o informales ofrecidas por la ciudad de La Paz. Cuando se trata de trabajadores de construcción, los empleos y los lugares de actividad se encuentran esencialmente en el municipio paceño. Ocurre lo mismo con los empleos domésticos cuya oferta proviene de los barrios acomodados situados en

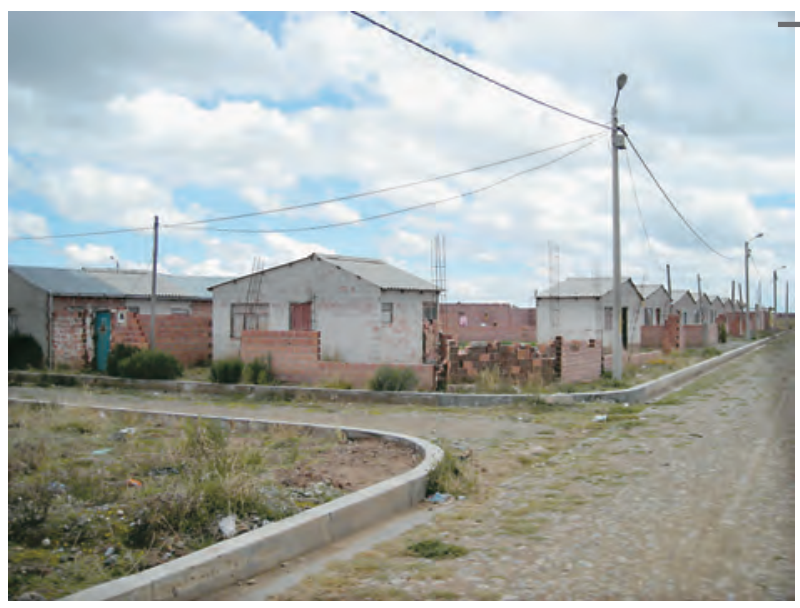

Figura 4 - Las viviendas de Cristal I, modestas, a menudo en un estado de semi abandono

Foto: S. Hardy, 2007 
la parte sur del municipio de La Paz. Muchos empleos también están vinculados a pequeñas actividades comerciales como puestos de venta en la calle, que proporcionan ingresos irregulares y poco remuneradores.

Pero los barrios Cristal I, Cristal II y Palermo están situados a más de una hora en bus de la Plaza Pérez Velazco, nudo de transporte al norte del centro de La Paz. La lejanía de La Paz representa por lo tanto un problema para los damnificados reubicados en estos tres barrios de El Alto. Además del tiempo de transporte, este desplazamiento de ida y vuelta representa un gasto de por lo menos 6 Bolivianos 11 diarios en 2008. Hay que contar, desde la Plaza Pérez Velazco, 3 Bolivianos más de ida y vuelta para llegar a la parte sur del municipio de La Paz, donde están concentradas la mayoría de las oportunidades de trabajo. Ahora bien, un albañil gana a menudo entre 20 a 30 Bolivianos diarios, cuando tiene la suerte de encontrar un trabajo. Por lo tanto, estos gastos diarios de desplazamiento pueden representar rápidamente para los reubicados entre el tercio y la mitad de los ingresos de un adulto activo. Lógicamente, esta situación no es soportable para las familias. Es por esta razón que la mayoría ha decidido regresar a vivir al municipio de La Paz para responder a ciertas prioridades (cercanía de las ofertas de trabajo, etc.) con el riesgo de exponerse a futuros daños de origen natural.

Estas decisiones muestran que no se trata necesariamente de un desconocimiento de la exposición al daño por parte de la población. Pero otras prioridades están por encima y permiten relativizar el riesgo: cercanía del lugar de trabajo, de establecimientos médicos y educacionales considerados de buen nivel, de mercados de abastecimiento barato, etc. Igualmente, la población autoevalúa su vulnerabilidad frente a la pérdida de acceso a ciertos elementos del funcionamiento urbano y, a partir de esto, jerarquiza su elección de exposición al riesgo (Nathan, 2008: 348-350; Nathan, 2009). Se puede hablar de elementos esenciales cuya pérdida representa un riesgo.

Por lo tanto, los efectos de la reubicación deben considerarse caso por caso. Por un lado, los damnificados que se quedaron en el lugar de reubicación por lo general son familias en que los padres de familia se ausentan de los hogares durante toda la semana para ir a trabajar al otro extremo de la ciudad. Esta situación no es excepcional y no es específica de los reubicados. Ha sido frecuentemente observada en muchas familias alteñas y se debe al costo elevado del transporte en relación al salario (Poupeau, 2007: 225). En cambio, otros damnificados abandonaron completamente los barrios de reubicación, sin ni siquiera esperar obtener un beneficio alquilando la vivienda. Se concluye que existe al menos un problema vinculado a la desarticulación entre el lugar de residencia, los lugares de actividad y el costo del transporte, y que este no fue anticipado por los actores de la reubicación, lo que explica ciertos fracasos.

Por otro lado, la ausencia de seguimiento de la reubicación de los damnificados por los principales actores institucionales no permite obtener lecciones útiles para pensar una política eficaz de reubicación. Evidentemente, la Comisión de

${ }^{11}$ La tasa de cambio promedio euros/bolivianos en 2008: $1 €=8$ bolivianos. 
damnificados es responsable del seguimiento institucional de los reubicados que se comprometieron a ocupar durante al menos 5 años su nueva vivienda. La Comisión puede efectuar verificaciones, eventualmente quitar la vivienda al beneficiario en caso de no respeto de las cláusulas. Sin embargo, en la práctica, la Comisión es constituida para cada gestión de crisis y desaparece a su término. Por lo tanto, carece de medios para efectuar cualquier seguimiento de los damnificados reubicados. Esto prueba que las autoridades consideran el riesgo como la exposición de la población a la amenaza: una vez que esta se soluciona, el riesgo desaparece y ya no es necesario preocuparse por ella.

Ahora bien, las prácticas de los damnificados reubicados ponen de manifiesto que esta exposición puede ser una elección pensada de las poblaciones frente a otros elementos considerados como esenciales (acceso a un trabajo, a escuelas, a centros de salud, etc.) cuya pérdida puede igualmente percibirse como un daño. El análisis de ciertos casos de reubicación muestra también una gran complejidad de las situaciones, que revela verdaderas estrategias de inserción urbana ocupando y valorizando varios espacios a la vez en la aglomeración.

\section{2. Traslados temporales para disminuir la vulnerabilidad}

Un trabajo de campo realizado en 2007 permitió la observación de prácticas más complejas de parte de los damnificados reubicados (Combaz, 2007). Incluso se puede hablar de verdaderas estrategias de inserción urbana, que aprovechan las complementariedades de los espacios.

El análisis parte de la desocupación de muchas viviendas en el barrio Cristal II, en particular las de los obreros de las fábricas de ladrillo de Alto Llojeta. Se constata también la reocupación de los terrenos situados en la parte occidental del municipio de La Paz, en Llojeta, por parte de los damnificados del deslizamiento de terreno del 4 de marzo de 2003, reubicados en Cristal II. Llojeta es su lugar de trabajo. Esta situación es ilegal ya que el procedimiento de reubicación aceptado por los damnificados indujo a un intercambio de actas de propiedad entre los terrenos de Llojeta y las viviendas en El Alto. Por lo tanto, los terrenos de Alto Llojeta son la propiedad de la municipalidad paceña, que los ha considerado inhabitables a causa de su exposición a amenazas de origen natural.

Las entrevistas realizadas con los obreros de las fábricas de ladrillos de Llojeta han permitido comprender esta reocupación. Al destruir su vivienda, el deslizamiento de terreno ha provocado la pérdida de sus ingresos provenientes de la fabricación de ladrillos. Entonces ellos decidieron recrear una cooperativa y, con la ayuda de la ONG Caritas Bolivia y el acuerdo de las autoridades municipales, volvieron a comprar el pedazo de terreno inicialmente cedido para instalar una nueva fábrica. Pero muy rápidamente el factor distancia entre el lugar de residencia y el lugar de actividades, correlacionado al costo del transporte, volvió insostenible la situación, dejando aparecer dos casos. 
- En el primer caso, el obrero de la fábrica de ladrillos trabaja durante la semana en Llojeta, y regresa el sábado a Cristal II. El pasa así más del 85 \% de su tiempo en Llojeta, viviendo en dormitorios construidos alrededor de la fábrica. Cabe preguntarse si su residencia principal no se sitúa finalmente más en La Paz que en El Alto. Sin embargo, en este ejemplo, la familia se queda en Cristal II, lo que hace pensar que sólo un miembro de la familia ejerce una forma de reapropiación parcial del barrio. Por otro lado, el trabajador no rehabilita su terreno afectado por el desastre ya que él vive en la fábrica. Esta ambigüedad explica por qué las autoridades han aceptado la situación: salvaguarda su concepto de gestión de los riesgos. Sobre todo, aquí se dibuja una estrategia temporal de los usos de los espacios, que responde a las necesidades de la vida cotidiana de algunas familias damnificadas.

- En un segundo ejemplo, se observa una reapropiación mucho más clara del espacio de Llojeta por algunos damnificados reubicados, confirmada por el hecho que algunas de las casas de Cristal II parecen desocupadas o sirven de depósito de ladrillos. Las entrevistas pusieron en evidencia otras estrategias espacio-temporales. Algunas familias de damnificados parecen haber integrado la exposición a la amenaza de un deslizamiento de terreno durante la temporada de lluvias en Llojeta, y por esta razón han elaborado una estrategia de uso de los espacios en función de las temporadas. Permanecen en Cristal II durante la temporada de lluvias, que corresponde también a un largo periodo de vacaciones escolares, para reducir su exposición al daño. Y el resto del año las familias residen en Llojeta para acumular ingresos, permitir a los niños el acceso a una escuela considerada de mejor calidad que en El Alto, y acceder más fácilmente a los servicios médicos. Por lo tanto, en este caso hay una real reapropiación del barrio de origen al volver a vivir en los terrenos dañados por el evento de marzo de 2003. Este caso prueba, una vez más, que la vivienda representa múltiples elementos esenciales para la población urbana más pobre (De Soto, 2000). Se trata de un lugar de residencia, que permite al mismo tiempo, en muchos casos, una actividad de orden familiar generadora de ingresos (fábrica de ladrillos) y acceso a algunos servicios. Su situación en la ciudad facilita, por la misma ocasión, un mejor anclaje de sus residentes a la ciudad y, por lo tanto, su reivindicación de la urbanidad (acceso al sistema de salud, acceso al sistema educativo).

Ahora bien, estos factores no fueron tomados en cuenta en absoluto por las autoridades en el momento de la elección de los lugares de reubicación, mientras que para la población sí eran considerados en su evaluación de la vulnerabilidad. Entre el limitado abanico de opciones que puede elegir, su gestión de los riesgos implica a menudo la aceptación de manera individual de una mayor exposición a la amenaza, pero con el objetivo de reducir su vulnerabilidad dándoles acceso a algunos espacios de La Paz y a sus servicios e infraestructuras. A fin de cuentas, la reflexión hecha por una gran mayoría de la población sobre sus opciones limitadas no es contemplada en absoluto por las autoridades, las cuales continúan pensando en la gestión de los riesgos esencialmente como la reducción de la exposición a la amenaza, además con un balance discutible (Hardy, 2009). 


\section{3. Los elementos esenciales y la gestión de las crisis}

Cuando los damnificados decidieron mantenerse en el lugar de la reubicación definitiva, no les fue fácil volver a empezar sus vidas en su nuevo barrio. Su llegada creó tensiones debido a la categorización de los residentes, consecuencia de las diferentes modalidades de acceso a la vivienda12.

Al principio, los barrios Cristal I, Cristal II y Palermo habían sido erigidos para acoger a los beneficiarios del PNVS desde 1999, así como a otras familias deseosas de acceder a la propiedad. Sus modalidades de acceso a las viviendas han diferido enormemente con las de las poblaciones damnificadas.

En efecto, los beneficiarios del PNVS compraron sus viviendas con ayuda de un préstamo bonificado garantizado por el Estado con una duración de 20 años. Además de la compra de la vivienda, pagaron los derechos de conexión a los servicios básicos, cuyo costo es bastante alto: 155 dólares para la red de agua potable por ejemplo, es decir el equivalente de tres salarios mínimos mensuales (García Linera, 2006). Los ingresos de numerosas familias de la aglomeración paceña no permiten pagar estos gastos. Además muchas de ellas optaron por invertir en la adquisición de una vivienda y postergar el acceso a los servicios urbanos. Es el caso de muchos beneficiarios del PNVS.

En el caso de los damnificados, se trata de una operación de reubicación por intercambio de actas de propiedad. Por lo tanto, son automáticamente propietarios de la vivienda que reciben, ya que hicieron el esfuerzo de compra de la vivienda anterior destruida por el desastre. Por la misma razón, su vivienda también está provista de una conexión a los servicios básicos.

Estas distinciones entre residentes tienen serias consecuencias. La participación en la vida del barrio de los beneficiarios de una operación de reubicación es, aunque no menos importante, al menos de diferente naturaleza. Por ejemplo, ellos participan menos en las asambleas de barrio para movilizaciones colectivas en torno a la reivindicación de las conexiones a los servicios básicos que ya tienen, mientras que los beneficiarios del PNVS y las otras familias de propietarios se movilizan frecuentemente para hacer presión sobre las autoridades políticas y tratar de obtener, sino las conexiones en forma gratuita, al menos una reducción de su costo. Esta menor participación en la colectividad es mal percibida por los beneficiarios del PNVS y crea algunas tensiones (como palabras despreciativas) entre habitantes. Estas diferencias entre los residentes se acentúan más aún por la división generada por la estructuración de los barrios. Los espacios de los beneficiarios del PNVS y otro residentes se diferencian mucho de los espacios de los damnificados beneficiarios de una reubicación, sea cual fuera el barrio analizado (fig. 5). A este nivel, la agrupación de los damnificados por desastre es muy visible: en Cristal II los damnificados de Llojeta están agrupados en el límite del barrio de los damnificados, mientras que los de Kupini, por ejemplo, están agrupados en

12 Ver también el articulo de R. D'Ercole, R. Cavagnoud, M. Morel y P. Vernier, en este volumen, pp. 647-681. 


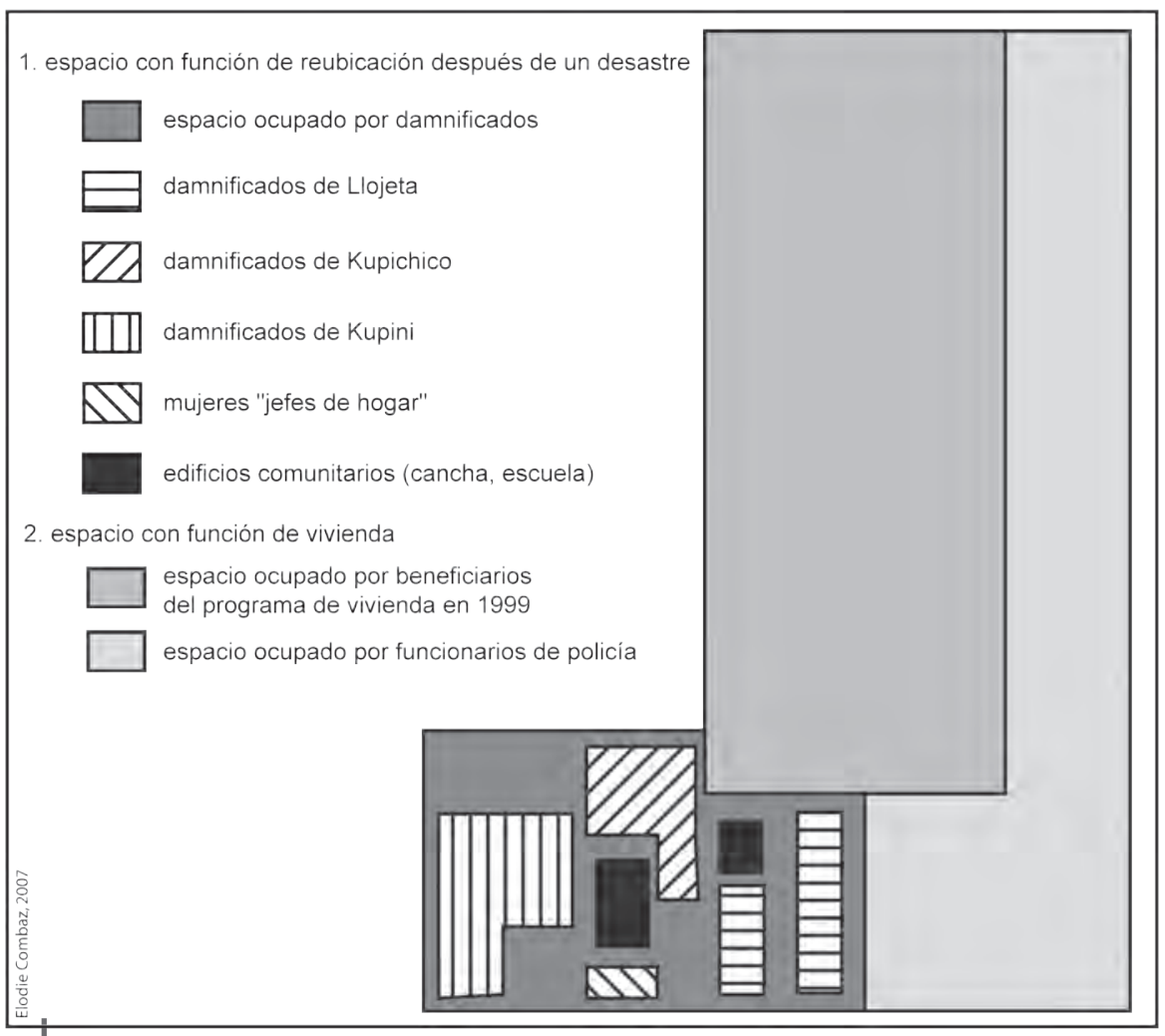

Figura 5 - Modelación del barrio Cristal II

la entrada del barrio. La organización espacial parece responder así a una lógica cronológica en el transcurso de la gestión de las crisis por parte de las autoridades, y crea un sentimiento identitario fundado en el evento catastrófico.

La organización del barrio no es el único factor explicativo de la división socioespacial observada. Las intervenciones posteriores a la reubicación también han podido favorecer las diferenciaciones entre damnificados. Por ejemplo, la Cruz Roja boliviana intervino con un programa de mejora de las condiciones de alojamiento de los damnificados reubicados del barrio Cristal I.

Las viviendas inicialmente entregadas, construidas sobre un terreno de $200 \mathrm{~m}^{2}$, eran de una superficie de $18 \mathrm{~m}^{2}$, sea cual fuere el número de miembros de cada familia. Los damnificados manifestaron rápidamente su incomodidad al habitarlas. El programa de la Cruz Roja consistía en la ampliación de la superficie habitable de las viviendas. Sin embargo, a causa del origen de la financiación —una donación de la Cruz Roja alemana en favor de los damnificados de la granizada del 19 de febrero de 2002- solo fue destinado a 68 familias seleccionadas en función de este criterio, cada una de las cuales recibió el equivalente de 4000 \$ US en 
materiales de construcción. Por lo tanto, algunas viviendas fueron modificadas y actualmente presentan una superficie habitable de $60 \mathrm{~m}^{2}$.

Esta intervención no solamente ha marcado una diferenciación entre algunos de los damnificados en comparación a los otros residentes del barrio, sino que explica también por qué algunos damnificados han decidido quedarse en el barrio de reubicación mientras que otros lo abandonaron. En efecto, las condiciones de vida de los beneficiarios de la ampliación de la vivienda mejoraron sensiblemente. En cambio, otros damnificados no beneficiarios se fueron al municipio de La Paz, insatisfechos por el desequilibrio entre las ventajas y los inconvenientes ofrecidos por la operación de reubicación. Ser beneficiario o no de las ayudas complementarias en los primeros tiempos después de la reubicación ha influido en el resultado de la operación. Estas ayudas han modificado la jerarquía de los elementos importantes: lo que las familias aceptan perder o no. Algunos damnificados aceptaron perder sus viviendas otorgadas después del desastre, ya que sus malas condiciones no fueron compensadas por un mejor acceso a espacios que ofrecen ventajas en términos de servicios y de infraestructuras, y que disminuyen ciertos factores de vulnerabilidad. En cambio, cuando las condiciones de la vivienda otorgada después del desastre mejoraron sensiblemente en comparación a la vivienda perdida, los elementos esenciales se distribuyeron nuevamente, a favor de una reducción de la vulnerabilidad.

\section{CONCLUSIÓN}

Al no anticipar el evento, la municipalidad paceña tuvo que manejar a tientas la crisis provocada por la granizada del 19 de febrero de 2002, tanto en fase de emergencia como en fase de recuperación. Para numerosos damnificados, el retorno a la normalidad estuvo vinculado a un muy largo proceso de reconstrucción. A pesar de ello, las autoridades a cargo de la crisis fueron capaces de realizar un análisis retrospectivo sobre sus tres diferentes fases y se dieron cuenta de la necesidad de anticipar las futuras crisis.

La organización concebida durante la emergencia de la gestión de crisis ha dado pruebas de sus aptitudes bajo presión y ha sido institucionalizada, y desde entonces se reajusta en la medida de nuevas experiencias. Aunque todavía quedan muchos problemas por solucionar, la municipalidad paceña parece ser la mejor preparada de Bolivia para manejar crisis relacionadas a eventos de origen natural. Con la realización del análisis retrospectivo ha mejorado considerablemente una de las herramientas de intervención política sobre la gestión de los riesgos: el de la gestión de las emergencias y de la recuperación.

Sin embargo, los límites de esta capacidad de gestión de crisis son evidentes, sobre todo en fase de reconstrucción. Las autoridades municipales han manejado eventos cuya magnitud ha justificado que fueran declarados como catástrofes nacionales por los gobiernos bolivianos sucesivos. Este reconocimiento ha activado ayudas nacionales y eventualmente internacionales que la municipalidad ha canalizado, 
principalmente en beneficio de la reubicación de los damnificados (compra de terrenos y construcción de viviendas). Gracias a este apoyo la municipalidad ha intervenido en la gestión del riesgo disminuyendo la exposición de los damnificados al daño de origen natural.

No obstante, el análisis de eventos de menor magnitud que afectaron al municipio paceño y dejaron damnificados sin vivienda, ha dado otra imagen de las capacidades de las autoridades locales a gestionar la reconstrucción (Combaz, 2007). Sin declaración de desastre nacional que les de acceso a la base territorial de los municipios vecinos (a través del otorgamiento de fondos), las autoridades paceñas tuvieron que administrar unos escasos terrenos urbanizables, no expuestos a amenazas, en los límites de su territorio. Por lo tanto, fueron incapaces de proponer un procedimiento de reubicación definitivo a los damnificados, muchos de los cuales, se resignaron a elegir una reubicación ilegal por necesidad.

Detrás de este problema se presenta el de la gobernanza de la aglomeración de La Paz. Al estar compuesta por varias municipalidades autónomas, los procedimientos — de tipo intercomunalidad del sistema francés- para resolver problemas en común no existen. Esta ausencia fragiliza al conjunto de la aglomeración puesto que debe enfrentar desafíos impuestos por la competencia de las otras aglomeraciones bolivianas, en primer lugar la de Santa Cruz de la Sierra.

Por otro lado, los procedimientos de reubicación de los damnificados, necesarios para un retorno a la normalidad, no son satisfactorios del todo. En primer lugar porque solo están dirigidos a los propietarios, dejando de lado a los damnificados inquilinos. Y en segundo lugar porque incluso para los damnificados reubicados la solución aparece restringida debido a que no están contempladas las condiciones socioeconómicas ni las aspiraciones de las personas. Estas fallas explican claramente la razón de los retornos hacia los lugares de origen, a pesar de estar expuestos a la amenaza, de las fuertes diferenciaciones socio-espaciales generadas en los mismos barrios y de las estrategias de ocupación de los espacios. Estas últimas prueban el dinamismo de los damnificados cuando se trata de sobrevivir, estableciendo una jerarquía de elementos esenciales frente al riesgo. Esto demuestra nuevamente que el riesgo traduce el hecho de poder perder lo que es importante par uno (D'Ercole \& Metzger, 2004).

Partiendo de esta definición del riesgo, las autoridades estarían en condiciones de considerar que para numerosas poblaciones, el riesgo reside menos en la exposición a las amenazas que en la pérdida de accesibilidad a ciertos espacios y a sus elementos: infraestructuras y servicios. Por lo tanto, sus políticas de gestión de los riesgos serían más eficaces porque estarían en conformidad con las prácticas de gestión de los riesgos de sus administrados. 
Experiencias paceñas en la gestión de crisis y de la vulnerabilidad

\section{Referencias citadas}

COMBAZ, E., 2007 - Le relogement des populations sinistrées de la municipalité de La Paz. Contextes, évolutions, et impacts des pratiques de relogement de trois catastrophes : la granizada (2002), Llojeta (2003) et German Jordan (1997), 107 pp.; Aix-enProvence: mémoire de Master 1 de Géographie, mention Sciences géographiques, spécialité Mondialisation et développement, Aix-Marseille 1.

DAUPHINE, A. \& PROVITOLO, D., 2007 - La résilience : un concept pour la gestion des risques. Annales de géographie, $\mathbf{n} .^{\circ}$ 654: 115-125.

D'ERCOLE, R. \& METZGER, P., 2004 - La vulnerabilidad del Distrito Metropolitano de Quito, 496 pp.; Quito: MDMQ/IRD.

D'ERCOLE, R. \& METZGER, P., 2009 - Enjeux territoriaux et vulnérabilité : une approche opérationnelle. In: Risques et environnement : recherches interdisciplinaires sur la vulnérabilité des sociétés (A. Peltier \& S. Becerra, eds.): 391-402; París: Éditions L'Harmattan.

DE SOTO, H., 2000 - The mystery of capital: why capitalism triumphs in the West and fails everywhere else, 275 pp.; New York: Basic Books.

GMLP, 2007 - Catálogo de instrumentos en gestión municipal para la reducción de riesgos y preparación ante emergencias, 74 pp.; La Paz: PNUD.

HARDY, S., 2009 - Explorer la construction de la résilience. Expériences de recherche à La Paz. In: Risques et environnement : recherches interdisciplinaires sur la vulnérabilité des sociétés (A. Peltier \& S. Becerra, eds.): 469-482; París: Éditions L'Harmattan.

NATHAN, F., 2008 - Risk Perception, Risk Management and Vulnerability to Landslides in the hill slopes in the city of La Paz, Bolivia. A Preliminary Statement. Disasters, 32 (3): 337-357.

NATHAN, F., 2009 - Comprendre le risque et la vulnérabilité. Une perspective de sciences sociales à propos des risques de glissement de terrain à La Paz, Bolivie. In: Risques et environnement : recherches interdisciplinaires sur la vulnérabilité des sociétés (A. Peltier \& S. Becerra, eds.): 117-128; París: Éditions L'Harmattan.

O'HARE, G. \& RIVAS, S., 2005 - The landslide hazard and human vulnerability in La Paz City, Bolivia. The Geographical Journal, vol. 171, n. ${ }^{\circ}$ 3: 239-258.

PELLING, M., 2003 - The vulnerability of cities-natural disasters and social resilience, James/ Earthscan, 256 pp.; Londres.

PIGEON, P., 2005 - Géographie critique des risques, 217 pp.; París: Economica/ Anthropos.

POUPEAU, F., 2007 - Movilizaciones políticas y gestión de la penuria de agua en los distritos populares de El Alto, Bolivia. In: Después de las guerras del agua, movilizaciones políticas (C. Crespo \& S. Spronk, eds.): 209-235; La Paz: CESU-UMSS, Plural editores.

REVET, S., 2007 - Anthropologie d'une catastrophe. Les coulées de boue de 1999 au Venezuela, 366 pp.; París: Presses Sorbonne Nouvelle.

VILLEGAS, S. (ed.), 2002 - Memoria del «Martes Negro». iEmergencia por granizada en La Paz!, 59 pp.; La Paz: GMLP, Comisión episcopal de pastoral social CARITAS. 